\title{
HOLOMORPHIC FLEXIBILITY PROPERTIES OF SPACES OF ELLIPTIC FUNCTIONS
}

\author{
DAVID BOWMAN
}

(Received 17 December 2016; first published online 2 March 2017)

2010 Mathematics subject classification: primary 32H02; secondary 32E10, 58B 12.

Keywords and phrases: Oka manifold, mapping space, elliptic function, complex space, several complex variables.

Let $X$ be an elliptic curve and $\mathbb{P}$ the Riemann sphere. Since $X$ is compact, it is a deep theorem of Douady that the set $O(X, \mathbb{P})$ consisting of holomorphic maps $X \rightarrow \mathbb{P}$ admits a complex structure. If $R_{n}$ denotes the set of maps of degree $n$, then Namba has shown for $n \geq 2$ that $R_{n}$ is a $2 n$-dimensional complex manifold. We study holomorphic flexibility properties of the spaces $R_{2}$ and $R_{3}$.

Firstly, we show that $R_{2}$ is homogeneous and hence an Oka manifold. Secondly, we present our main theorem, that there is a six-sheeted branched covering space of $R_{3}$ that is an Oka manifold. It follows that $R_{3}$ is $\mathbb{C}$-connected and dominable. We show that $R_{3}$ is Oka if and only if $\mathbb{P}_{2} \backslash C$ is Oka, where $C$ is a cubic curve that is the image of a certain embedding of $X$ into $\mathbb{P}_{2}$.

We investigate the strong dominability of $R_{3}$ and show that if $X$ is not biholomorphic to $\mathbb{C} / \Gamma_{0}$, where $\Gamma_{0}$ is the hexagonal lattice, then $R_{3}$ is strongly dominable.

As a Lie group, $X$ acts freely on $R_{3}$ by precomposition by translations. We show that $R_{3}$ is holomorphically convex and that the quotient space $R_{3} / X$ is a Stein manifold.

We construct an alternative six-sheeted Oka branched covering space of $R_{3}$ and prove that it is isomorphic to our first construction in a natural way. This alternative construction gives us an easier way of interpreting the fibres of the branched covering map.

The full thesis is available at https://arxiv.org/abs/1609.07184.

DAVID BOWMAN, School of Mathematical Sciences,

University of Adelaide, Adelaide, South Australia 5005, Australia

e-mail: davidbowman@netspace.net.au

Thesis submitted to the University of Adelaide in September 2016; degree approved on 6 December 2016, principal supervisor Finnur Larusson and associate supervisor Nicholas Buchdahl.

(C) 2017 Australian Mathematical Publishing Association Inc. 0004-9727/2017 \$16.00 